\title{
EXISTENCE OF POSITIVE SOLUTIONS FOR A COUPLED SYSTEM OF NONLINEAR BOUNDARY VALUE PROBLEMS OF FRACTIONAL ORDER WITH INTEGRAL BOUNDARY CONDITIONS
}

\author{
Moses B. Akorede ${ }^{1}$, Peter O. Arawomo ${ }^{2} \S$ \\ ${ }^{1,2}$ Department of Mathematics \\ University of Ibadan \\ Ibadan, NIGERIA
}

\begin{abstract}
In this work, we discuss the existence of positive solutions for a coupled system of nonlinear boundary value problems of fractional order with integral boundary conditions.

The existence result is obtained by means of Krasnosel'skii fixed-point theorem in a cone.
\end{abstract}

AMS Subject Classification: 2010, 34A08, 34B15

Key Words: fractional derivatives, integral boundary conditions, positive solutions, coupled system, cone

\section{Introduction}

In this paper, we study the existence of positive solutions to the following coupled system of nonlinear boundary value problems of fractional order

Received: March 8, 2017

(C) 2017 Academic Publications

${ }^{\S}$ Correspondence author 


$$
\left.\begin{array}{r}
D^{\alpha} u(t)+w(t) f(t, v(t))=0, \quad t \in(0,1), \\
D^{\alpha} v(t)+z(t) g(t, u(t))=0, \quad t \in(0,1), \\
u(0)=0, \quad \gamma u(1)+\beta u^{\prime}(1)=\int_{0}^{1} p(t) u(t) d t, \\
v(0)=0, \quad \gamma v(1)+\beta v^{\prime}(1)=\int_{0}^{1} p(t) v(t) d t,
\end{array}\right\}
$$

where $1<\alpha \leq 2, D^{\alpha}$ is the standard Riemann-Liouville fractional derivative, $w, z \in C\left([0,1], \mathbb{R}^{+}\right), p \in \mathcal{L}^{1}[0,1]$ is non-negative, $\gamma, \beta \in(0,1)$ and $f, g \in$ $C\left([0,1] \times \mathbb{R}^{+}, \mathbb{R}^{+}\right)$.

In recent years, the study of positive solutions for nonlinear fractional differential equations and coupled systems has received much attention from many authors, see [1 - 2, 4, 6 - 9, 11 - 14, 16 - 17, 21 - 28, 30 - 33] and the references cited therein for details.

In [23], the authors studied the existence and uniqueness of positive solutions to the following nonzero boundary value problem (BVP for short) for a coupled system of nonlinear fractional differential equations:

$$
\left.\begin{array}{r}
D^{\alpha} u(t)+f(t, v(t))=0, \quad t \in(0,1), \\
D^{\beta} v(t)+f(t, u(t))=0, \quad t \in(0,1), \\
u(0)=0, \quad u(1)=a u(\xi), \\
v(0)=0, \quad v(1)=b v(\xi),
\end{array}\right\}
$$

where $1<\alpha<2,1<\beta<2,0 \leq a, b \leq 1,1<\xi<2, D^{\alpha}$ and $D^{\beta}$ are the standard Riemann-Liouville fractional derivatives.

In [14], the authors established the existence of a positive solution for the 
singular fractional differential equations with integral boundary conditions

$$
\left.\begin{array}{l}
{ }^{c} D^{p} u(t)=\lambda h(t) f(t, u(t)), \quad t \in(0,1), \\
u(0)-a u(1)=\int_{0}^{1} g_{0}(s) u(s) d s, \\
u^{\prime}(0)-b^{c} D^{q} u(1)=\int_{0}^{1} g_{1}(s) u(s) d s, \\
u^{\prime \prime}(0)=u^{\prime \prime \prime}(0)=\cdots=u^{(n-1)}(0)=0,
\end{array}\right\}
$$

where ${ }^{c} D^{p}$ is the Caputo fractional derivative, $n \geq 3$ is an integer, $p \in(n-1, n)$, $0<q<1,0<a<1,0<b<\Gamma(2-q)$ are real numbers.

Further, the authors in [10] studied the existence of positive solutions to the following integral boundary value problem of nonlinear fractional differential equation

$$
\left.\begin{array}{r}
D^{\alpha} x(t)+g(t) f(t, x)=0, \quad t \in(0,1), \\
x(0)=0, x^{\prime}(1)=\int_{0}^{1} h(t) x(t) d t,
\end{array}\right\}
$$

where $1<\alpha \leq 2, D^{\alpha}$ is the standard Riemann-Liouville fractional derivative and $h \in \mathcal{L}^{1}[0,1]$ is non-negative.

Inspired by the works in $[10,14,23]$, we consider in this paper the existence of positive solutions to the BVP (1). Many papers have dealt with coupled systems of nonlinear fractional differential equation with integral boundary conditions. However, to the best of our knowledge, the existence of positive solutions to the BVP (1) has not been discussed. In this paper, we consider an integral boundary condition which is very different from those in $[1-4,10-12,16,21$, 23, 26, 29 - 33]. Our approach is based on compact integral operator and the application of Krasnosel'skii fixed-point theorem in a cone.

For this work, we make the following assumptions:

$C_{1} . f:[0,1] \times[0, \infty) \longrightarrow[0, \infty)$ and $g:[0,1] \times[0, \infty) \longrightarrow[0, \infty)$ are continuous.

$C_{2} . w:[0,1] \longrightarrow[0, \infty)$ and $z:[0,1] \longrightarrow[0, \infty)$ are continuous and $w(t) \neq$ $0, z(t) \neq 0$ on any subinterval of $[0,1]$.

The paper is organized as follows: In Section 2, some basic definitions, preliminary results and properties of the Green function used are presented. Finally, our existence result is stated and proved in Section 3. 


\section{Preliminary Results}

In this section, we give some basic definitions and lemmas from the theory of fractional calculus which will be needed in the sequel. Moreover, we give the expression of Green's function associated with the BVP (1).

Definition 2.1. ([5, 19, 20]) The Riemann-Liouville fractional integral of order $\alpha>0$ for a given continuous function $f:(0, \infty) \longrightarrow \mathbb{R}$ is defined to be

$$
I_{0^{+}}^{\alpha} f(t)=\frac{1}{\Gamma(\alpha)} \int_{0}^{t}(t-s)^{\alpha-1} f(s) d s,
$$

provided the right side is pointwise defined on $(0, \infty)$.

Definition 2.2. ([5, 19, 20]) The Riemann-Liouville fractional derivative of order $\alpha>0$ for a given continuous function $f:(0, \infty) \longrightarrow \mathbb{R}$ is defined to be

$$
D_{0^{+}}^{\alpha} f(t)=\frac{1}{\Gamma(n-\alpha)}\left(\frac{d}{d t}\right)^{n} \int_{0}^{t}(t-s)^{n-\alpha-1} f(s) d s,
$$

$n-1<\alpha \leq n$, provided the right side is pointwise defined on $(0, \infty)$, where $n=[\alpha]+1$ and $[\alpha]$ is the integer part of the number $\alpha$.

Lemma 2.3. ([18]) If $u \in C(0,1) \cap \mathcal{L}(0,1)$, then

$$
D^{\alpha} I^{\alpha} u(t)=u(t) .
$$

Lemma 2.4. ([5, 20]) Let $\alpha>0$ and $u \in C(0,1) \cap \mathcal{L}(0,1)$. Then the unique solution of $D^{\alpha} u(t)=0$ is given by

$$
u(t)=c_{1} t^{\alpha-1}+c_{2} t^{\alpha-2}+\cdots+c_{n} t^{\alpha-n},
$$

for $c_{i} \in \mathbb{R}$ and $i=1,2, \ldots, n$.

Lemma 2.5. ([5, 20]) Let $\alpha>0$ and $u, D^{\alpha} u \in C(0,1) \cap \mathcal{L}(0,1)$. Then

$$
\left.\begin{array}{r}
I^{\alpha} D^{\alpha} u(t)=u(t)+c_{1} t^{\alpha-1}+c_{2} t^{\alpha-2}+\cdots+c_{n} t^{\alpha-n}, \\
\text { for } c_{i} \in \mathbb{R} \text { and } i=1,2, \ldots, n, \quad n \geq \alpha .
\end{array}\right\}
$$


Lemma 2.6. Let $h \in C[0,1]$ and $\eta=\frac{1}{a_{0}} \int_{0}^{1} p(t) t^{\alpha-1} d t$. Then the unique solution of the BVP

$$
\left.\begin{array}{r}
D^{\alpha} u(t)+h(t)=0, \quad 0<t<1, \\
u(0)=0, \quad \gamma u(1)+\beta u^{\prime}(1)=\int_{0}^{1} p(t) u(t) d t
\end{array}\right\}
$$

is given by

$$
u(t)=\int_{0}^{1} G^{\star}(t, s) h(s) d s
$$

where

$$
\begin{gathered}
G^{\star}(t, s)=G_{1}(t, s)+G_{2}(t, s), \\
G_{1}(t, s)=\left\{\begin{array}{r}
\frac{1}{a_{0} \Gamma(\alpha)}\left[\beta(\alpha-1) t^{\alpha-1}(1-s)^{\alpha-2}+\gamma t^{\alpha-1}(1-s)^{\alpha-1}\right. \\
\left.-a_{0}(t-s)^{\alpha-1}\right], s \leq t, \\
\frac{1}{a_{0} \Gamma(\alpha)}\left[\beta(\alpha-1) t^{\alpha-1}(1-s)^{\alpha-2}+\gamma t^{\alpha-1}(1-s)^{\alpha-1}\right], \\
t \leq s,
\end{array}\right.
\end{gathered}
$$

and

$$
G_{2}(t, s)=\lambda_{0} G_{1}(t, s), \quad \lambda_{0}=\left(\frac{\eta}{1-\eta}\right), \quad 0<\eta<1
$$

Proof. By Lemma 2.5, the BVP (7) can be reduced to an equivalent integral equation

$$
\begin{aligned}
u(t) & =-I^{\alpha} h(t)+c_{1} t^{\alpha-1}+c_{2} t^{\alpha-2} \\
& =-\frac{1}{\Gamma(\alpha)} \int_{0}^{t}(t-s)^{\alpha-1} h(s) d s+c_{1} t^{\alpha-1}+c_{2} t^{\alpha-2} .
\end{aligned}
$$

By $u(0)=0$, we have $c_{2}=0$ and

$$
\begin{gathered}
u(t)=-\frac{1}{\Gamma(\alpha)} \int_{0}^{t}(t-s)^{\alpha-1} h(s) d s+c_{1} t^{\alpha-1} \\
u^{\prime}(t)=\frac{-(\alpha-1)}{\Gamma(\alpha)} \int_{0}^{t}(t-s)^{\alpha-2} h(s) d s+c_{1}(\alpha-1) t^{\alpha-2},
\end{gathered}
$$




$$
\begin{gathered}
\beta u^{\prime}(1)=-\frac{\beta(\alpha-1)}{\Gamma(\alpha)} \int_{0}^{1}(1-s)^{\alpha-2} h(s) d s+\beta(\alpha-1) c_{1}, \\
\gamma u(1)=-\frac{\gamma}{\Gamma(\alpha)} \int_{0}^{1}(1-s)^{\alpha-1} h(s) d s+\gamma c_{1} .
\end{gathered}
$$

Using the boundary condition $\gamma u(1)+\beta u^{\prime}(1)=\int_{0}^{1} p(t) u(t) d t$, we have

$$
\begin{gathered}
{[\beta(\alpha-1)+\gamma] c_{1}-\frac{\gamma}{\Gamma(\alpha)} \int_{0}^{1}(1-s)^{\alpha-1} h(s) d s-\frac{\beta(\alpha-1)}{\Gamma(\alpha)} \int_{0}^{1}(1-s)^{\alpha-2} h(s) d s} \\
=\int_{0}^{1} p(t) u(t) d t, \\
\Longrightarrow c_{1}=\frac{\gamma}{a_{0} \Gamma \alpha} \int_{0}^{1}(1-s)^{\alpha-1} h(s) d s+\frac{\beta(\alpha-1)}{a_{o} \Gamma \alpha} \int_{0}^{1}(1-s)^{\alpha-2} h(s) d s \\
+\frac{1}{a_{0}} \int_{0}^{1} p(t) u(t) d t
\end{gathered}
$$

where $a_{0}=[\beta(\alpha-1)+\gamma]>0$.

Putting (14) into (12), we have the unique solution of the BVP (7) to be:

$$
\begin{gathered}
u(t)=-\frac{1}{\Gamma(\alpha)} \int_{0}^{t}(t-s)^{\alpha-1} h(s) d s+\frac{\gamma}{a_{0} \Gamma(\alpha)} \int_{0}^{1} t^{\alpha-1}(1-s)^{\alpha-1} h(s) d s \\
+\frac{\beta(\alpha-1)}{a_{0} \Gamma(\alpha 0} \int_{0}^{1} t^{\alpha-1}(1-s)^{\alpha-2} h(s) d s+\frac{t^{\alpha-1}}{a_{0}} \int_{0}^{1} p(t) u(t) d t \\
=-\frac{1}{a_{0} \Gamma(\alpha)} \int_{0}^{t} a_{0}(t-s)^{\alpha-1} h(s) d s+\frac{\gamma}{a_{0} \Gamma(\alpha)} \int_{0}^{t} t^{\alpha-1}(1-s)^{\alpha-1} h(s) d s \\
+\frac{\gamma}{a_{0} \Gamma(\alpha)} \int_{t}^{1} t^{\alpha-1}(1-s)^{\alpha-1} h(s) d s+\frac{\beta(\alpha-1)}{a_{0} \Gamma(\alpha)} \int_{0}^{t} t^{\alpha-1}(1-s)^{\alpha-2} h(s) d s \\
+\frac{\beta(\alpha-1)}{a_{0} \Gamma(\alpha)} \int_{t}^{1} t^{\alpha-1}(1-s)^{\alpha-2} h(s) d s+\frac{t^{\alpha-1}}{a_{0}} \int_{0}^{1} p(t) u(t) d t \\
\Longrightarrow u(t)=\int_{0}^{1} G_{1}(t, s) h(s) d s+\frac{t^{\alpha-1}}{a_{0}} \int_{0}^{1} p(t) u(t) d t,
\end{gathered}
$$

where $G_{1}(t, s)$ is defined by $(9)$. 
Multiplying (15) by $p(t)$ and integrating from 0 to 1 , we have

$$
\begin{gathered}
\int_{0}^{1} p(t) u(t) d t=\int_{0}^{1} p(t) \int_{0}^{1} G_{1}(t, s) h(s) d s d t \\
\quad+\frac{1}{a_{0}} \int_{0}^{1} p(t) t^{\alpha-1} d t \cdot \int_{0}^{1} p(t) u(t) d t \\
\Longrightarrow \int_{0}^{1} p(t) u(t) d t-\eta \int_{0}^{1} p(t) u(t) d t=\int_{0}^{1} p(t) \int_{0}^{1} G_{1}(t, s) h(s) d s d t
\end{gathered}
$$

where

$$
\begin{gathered}
\eta=\frac{1}{a_{0}} \int_{0}^{1} p(t) t^{\alpha-1} d t \\
\Longrightarrow(1-\eta) \int_{0}^{1} p(t) u(t) d t=\int_{0}^{1} p(t) \int_{0}^{1} G_{1}(t, s) h(s) d s d t \\
\Longrightarrow \int_{0}^{1} p(t) u(t) d t=\frac{1}{1-\eta} \int_{0}^{1} p(t) \int_{0}^{1} G_{1}(t, s) h(s) d s d t .
\end{gathered}
$$

Put (16) into (15) and simplifying, we have

$$
\begin{gathered}
u(t)=\int_{0}^{1} G_{1}(t, s) h(s) d s+\frac{1}{a_{0}} \int_{0}^{1} p(t) t^{\alpha-1} d t \cdot \frac{1}{1-\eta} \int_{0}^{1} G_{1}(t, s) h(s) d s \\
=\int_{0}^{1} G_{1}(t, s) h(s) d s+\frac{\eta}{1-\eta} \int_{0}^{1} G_{1}(t, s) h(s) d s \\
=\int_{0}^{1}\left[G_{1}(t, s)+\frac{\eta}{1-\eta} G_{1}(t, s)\right] h(s) d s \\
\Longrightarrow u(t)=\int_{0}^{1} G^{\star}(t, s) h(s) d s
\end{gathered}
$$

where

$$
G^{\star}(t, s)=G_{1}(t, s)+G_{2}(t, s) \text { and } G_{1}(t, s), G_{2}(t, s)
$$

are defined by (9) and (10) respectively. This completes the proof.

Lemma 2.7. The function $G_{1}(t, s)$ defined by (9) is continuous and satisfies the following conditions:

(i) $G_{1}(t, s) \geq 0 \forall t, s \in[0,1]$ and $G_{1}(t, s)>0, \forall t, s \in(0,1)$;

(ii) $G_{1}(t, s) \leq G_{1}(s, s)=\frac{s^{\alpha-1}\left[\beta(\alpha-1)(1-s)^{\alpha-2}+\gamma(1-s)^{\alpha-1}\right]}{a_{0} \Gamma(\alpha)}$, for all $t, s \in[0,1]$; 
(iii) $\min _{\frac{1}{4} \leq t \leq \frac{3}{4}} G_{1}(t, s) \geq m(s) \max _{0 \leq t \leq 1} G_{1}(t, s)=m(s) G_{1}(s, s)$,

for $\frac{1}{4} \leq t \leq \frac{3}{4}, \quad s \in(0,1)$ and $0<m(s)<1$,

where $m(s) \in C\left((0,1), \mathbb{R}^{+}\right)$and

$$
m(s)=\left\{\begin{array}{l}
\frac{\left(\frac{3}{4}\right)^{\alpha-1}\left[\beta(\alpha-1)(1-s)^{\alpha-2}+\gamma(1-s)^{\alpha-1}\right]-a_{o}\left(\frac{3}{4}-s\right)^{\alpha-1}}{s^{\alpha-1}\left[\beta(\alpha-1)(1-s)^{\alpha-2}+\gamma(1-s)^{\alpha-1}\right]} s \in\left(0, \frac{3}{4}\right], \\
\frac{1}{(4 s)^{\alpha-1}}, s \in\left[\frac{3}{4}, 1\right) .
\end{array}\right.
$$

Proof.

(i) It is standard and omitted.

(ii) For $t \leq s$, we have

$$
\begin{aligned}
\frac{G_{1}(t, s)}{G_{1}(s, s)} & =\frac{\beta(\alpha-1) t^{\alpha-1}(1-s)^{\alpha-2}+\gamma t^{\alpha-1}(1-s)^{\alpha-1}}{\beta(\alpha-1) s^{\alpha-1}(1-s)^{\alpha-2}+\gamma s^{\alpha-1}(1-s)^{\alpha-1}} \\
& =\frac{t^{\alpha-1}\left[\beta(\alpha-1)(1-s)^{\alpha-2}+\gamma(1-s)^{\alpha-1}\right]}{s^{\alpha-1}\left[\beta(\alpha-1)(1-s)^{\alpha-2}+\gamma(1-s)^{\alpha-1}\right]} \\
& =\frac{t^{\alpha-1}}{s^{\alpha-1}} \leq 1 .
\end{aligned}
$$

Similarly, for $s \leq t$, we have $\frac{G_{1}(t, s)}{G_{1}(s, s)} \leq 1$.

Hence, $G_{1}(t, s) \leq G_{1}(s, s)=\frac{s^{\alpha-1}\left[\beta(\alpha-1)(1-s)^{\alpha-2}+\gamma(1-s)^{\alpha-1}\right]}{a_{0} \Gamma(\alpha)}$, for all $t, s \in[0,1]$.

(iii) The proof of (iii) is similar to that of Lemma 2.4 in [5] and so omitted.

Lemma 2.8. Suppose $0<\eta<1$. Then $G_{2}(t, s)$ defined by (10) is continuous and satisfies the following conditions:

(i) $G_{2}(t, s) \geq 0$ for all $t, s \in[0,1]$ and $G_{2}(t, s)>0$ for all $t, s \in(0,1)$.

(ii) $G_{2}(t, s) \leq \lambda_{0} G_{1}(s, s)$

$=\frac{\lambda_{0} s^{\alpha-1}\left[\beta(\alpha-1)(1-s)^{\alpha-2}+\gamma(1-s)^{\alpha-1}\right]}{a_{0} \Gamma(\alpha)}$,

$\forall t, s \in[0,1]$, where $\lambda_{0}=\left(\frac{\eta}{1-\eta}\right)>0$. 
Lemma 2.9. The Green function $G^{\star}(t, s)$ defined by $(8)$ is continuous and satisfies the following conditions:

(i) $G^{\star}(t, s) \geq 0$ for all $t, s \in[0,1]$ and $G^{\star}(t, s)>0$ for all $t, s \in(0,1)$;

(ii) $G^{\star}(t, s) \leq \sigma G_{1}(s, s)=\frac{\sigma s^{\alpha-1}\left[\beta(\alpha-1)(1-s)^{\alpha-2}+\gamma(1-s)^{\alpha-1}\right]}{a_{0} \Gamma(\alpha)}$,

$\forall t, s \in[0,1]$, where $\sigma=\left(1+\lambda_{0}\right)>0 ;$

(iii) $\min _{\frac{1}{4} \leq t \leq \frac{3}{4}} G^{\star}(t, s) \geq \sigma m(s) G_{1}(s, s)$, for $\frac{1}{4} \leq t \leq \frac{3}{4}, s \in(0,1)$.

Proof. (i) It is standard and omitted.

(ii) In view of Lemmas 2.7, 2.8 and equation (8), we have

$$
\begin{aligned}
G^{\star}(t, s) & =G_{1}(t, s)+G_{2}(t, s) \\
& =G_{1}(t, s)+\lambda_{0} G_{1}(t, s) \\
& \leq G_{1}(s, s)+\lambda_{0} G_{1}(s, s) \\
& \leq\left(1+\lambda_{0}\right) G_{1}(s, s) \\
& \leq \frac{\sigma s^{\alpha-1}\left[\beta(\alpha-1)(1-s)^{\alpha-2}+\gamma(1-s)^{\alpha-1}\right]}{a_{0} \Gamma(\alpha)}
\end{aligned}
$$

where $\sigma=\left(1+\lambda_{0}\right)>0$.

(iii) By the standard argument of [5], we have

$$
\max _{0 \leq t \leq 1} G_{1}(t, s)=G_{1}(s, s) \text { and } \max _{0 \leq t \leq 1} G_{2}(t, s)=\lambda_{0} G_{1}(s, s) .
$$

Also,

$$
\begin{aligned}
\max _{0 \leq t \leq 1} G^{\star}(t, s) & =\max _{0 \leq t \leq 1}\left[G_{1}(t, s)+G_{2}(t, s)\right], \\
& =\max _{0 \leq t \leq 1} G_{1}(t, s)+\max _{0 \leq t \leq 1} G_{2}(t, s), \\
& =G_{1}(s, s)+\lambda_{0} G_{1}(s, s), \\
& =\left(1+\lambda_{0}\right) G_{1}(s, s)=\sigma G_{1}(s, s) . \\
\min _{\frac{1}{4} \leq t \leq \frac{3}{4}} G^{\star}(t, s) & =\min _{\frac{1}{4} \leq t \leq \frac{3}{4}}\left[G_{1}(t, s)+G_{2}(t, s)\right], \\
\geq & \min _{\frac{1}{4} \leq t \leq \frac{3}{4}}\left[G_{1}(t, s)+\lambda_{0} G_{1}(t, s)\right], \\
\geq & \left(1+\lambda_{0}\right) \min _{\frac{1}{4} \leq t \leq \frac{3}{4}} G_{1}(t, s), \\
\geq &
\end{aligned}
$$


In view of Lemma 2.6, $(u, v) \in C[0,1] \cap \mathcal{L}^{1}[0,1]$ is a solution of the BVP $(1)$ if and only if $(u, v)$ solves the system of integral equations

$$
\left.\begin{array}{c}
u(t)=\int_{0}^{1} G^{\star}(t, s) w(s) f(s, v(s)) d s, \\
v(t)=\int_{0}^{1} G^{\star}(t, s) z(s) g(s, u(s)) d s,
\end{array}\right\}
$$

where $G^{\star}(t, s)$ is defined by $(8)$.

The system of integral equations (19) can be written as

$$
\begin{array}{r}
u(t)=\int_{0}^{1} G^{\star}(t, s) w(s) f\left(s, \int_{0}^{1} G^{\star}(s, r) z(r) g(r, u(r)) d r\right) d s \\
t \in(0,1) .
\end{array}
$$

Let $B^{*}=C[0,1]$ be a Banach space with norm $\|u\|=\max _{0 \leq t \leq 1}|u(t)|$. Define a cone $K_{*} \subset B^{*}$ by

$$
K_{*}=\left\{u \in B^{*}: u(t) \geq 0 \text { and } \min _{\frac{1}{4} \leq t \leq \frac{3}{4}} u(t) \geq m(s)\|u\| .\right\} .
$$

Define an integral operator $A: K_{*} \longrightarrow B^{*}$ by

$$
A u(t)=\int_{0}^{1} G^{\star}(t, s) w(s) f\left(s, \int_{0}^{1} G^{\star}(s, r) z(r) g(r, u(r)) d r\right) d s,
$$

for $u \in K_{*}$.

Lemma 2.10. ([3]) Let the operator $A$ be defined as in (21). Then $A$ : $K_{*} \longrightarrow K_{*}$ is completely continuous.

In view of the fixed point theory, the existence of positive solutions to the BVP (1) is equivalent to the existence of positive fixed points of the operator $A$ in the cone $K_{*}$. We state the Krasnosel'skii fixed-point theorem, as follows.

Theorem 2.11. ([15, 29]) Let $B^{*}$ be a Banach Space and $K_{*} \subset B^{*}$ be a cone in $B^{*}$. Assume $\Omega_{1}, \Omega_{2}$ are open subsets of $B^{*}$ such that $0 \in \Omega_{1}, \bar{\Omega}_{1} \subset \Omega_{2}$.

If $A: K_{*} \cap\left(\bar{\Omega}_{2} \backslash \Omega_{1}\right) \longrightarrow K_{*}$ is a completely continuous operator such that either

(i) $\|A u\| \leq\|u\|, u \in K_{*} \cap \partial \Omega_{1}$ and $\|A u\| \geq\|u\|, u \in K_{*} \cap \partial \Omega_{2}$, or

(ii) $\|A u\| \geq\|u\|, u \in K_{*} \cap \partial \Omega_{1}$ and $\|A u\| \leq\|u\|, \quad u \in K_{*} \cap \partial \Omega_{2}$,

then $A$ has a fixed point in $K_{*} \cap\left(\bar{\Omega}_{2} \backslash \Omega_{1}\right)$. 


\section{Existence Result}

In this section, we apply the fixed point Theorem 2.11 to establish the existence of positive solutions to the BVP (1).

Theorem 3.1. Assume conditions $C_{1}, C_{2}$ and the following hypotheses hold:

$\left(H_{1}\right)$ There exist positive constants $k_{1}, k_{2}$ and $e_{1}$ such that $f(t, u) \leq k_{1} u$ and $g(t, u) \leq k_{2} u$, for $(t, u) \in\left([0,1] \times\left[0, e_{1}\right]\right)$, where $k_{1}$ and $k_{2}$ satisfy

$$
\sigma k_{1} \int_{0}^{1} G_{1}(s, s) w(s) d s \leq 1 \text { and } \sigma k_{2} \int_{0}^{1} G_{1}(r, r) z(r) d r \leq 1
$$

respectively.

$\left(H_{2}\right)$ There exist positive constants $\lambda_{1}, \lambda_{2}$ and $e_{2}$ such that $f(t, u) \geq \lambda_{1} u$ and $g(t, u) \geq \lambda_{2} u$, for $(t, u) \in\left(\left[\frac{1}{4}, \frac{3}{4}\right] \times\left(0, e_{2}\right]\right)$ with $e_{1}>e_{2}$, where $\lambda_{1}$ and $\lambda_{2}$ satisfy

$$
\lambda_{1} \int_{1 / 4}^{3 / 4} \sigma G_{1}\left(\frac{1}{2}, s\right) w(s) d s \geq 1 \text { and } \lambda_{2} \int_{1 / 4}^{3 / 4} \sigma m(r) G_{1}(r, r) z(r) d r \geq 1
$$

respectively.

Then the BVP (1) has at least one positive solution $u(t)$ in the cone $K_{*}$.

Proof. Let $u \in K_{*}$ with $\|u\|=e_{1}$. By hypothesis $\left(H_{1}\right)$, we have $0 \leq u \leq e_{1}$ and

$$
A u(t)=\int_{0}^{1} G^{\star}(t, s) w(s) f\left(s, \int_{0}^{1} G^{\star}(s, r) z(r) g(r, u(r)) d r\right) d s
$$




$$
\begin{aligned}
&\|A u\| \leq \int_{0}^{1} G^{\star}(t, s) w(s) d s \cdot k_{1} \int_{0}^{1} G^{\star}(s, r) z(r) g(r, u(r)) d r \\
& \leq \sigma \int_{0}^{1} G_{1}(s, s) w(s) d s \cdot \sigma k_{1} \int_{0}^{1} G_{1}(r, r) z(r) g(r, u(r)) d r \\
& \leq \sigma \int_{0}^{1} G_{1}(s, s) w(s) d s \cdot \sigma k_{1} \int_{0}^{1} G_{1}(r, r) z(r) \cdot k_{2} u d r \\
& \leq \sigma k_{1} \int_{0}^{1} G_{1}(s, s) w(s) d s \cdot \sigma k_{2} \int_{0}^{1} G_{1}(r, r) z(r) \cdot u d r \\
& \leq \sigma k_{1} \int_{0}^{1} G_{1}(s, s) w(s) d s \cdot \sigma k_{2} \int_{0}^{1} G_{1}(r, r) z(r) \cdot c_{1} d r \\
&=\sigma k_{1} \int_{0}^{1} G_{1}(s, s) w(s) d s \cdot \sigma k_{2} \int_{0}^{1} G_{1}(r, r) z(r)\|u\| d r . \\
& \Longrightarrow\|A u\| \leq\|u\| .
\end{aligned}
$$

If we set $\Omega_{1}=\left\{u \in B^{*}:\|u\|<\rho_{1}\right\}$, then $\|A u\| \leq\|u\|$, for $u \in\left(K_{*} \cap \partial \Omega_{1}\right)$.

Next, let $u \in K_{*}$ with $\|u\|=e_{2}$. Then for $\frac{1}{4} \leq t \leq \frac{3}{4}$, we have

$$
u(t) \geq \min _{\frac{1}{4} \leq t \leq \frac{3}{4}} u(t) \geq m(s)\|u\|=m(s) e_{2},
$$

whenever $m(s) e_{2} \leq u \leq e_{2}$.

By hypothesis $\left(H_{2}\right)$, we have:

$$
\begin{aligned}
A u\left(\frac{1}{2}\right) & =\int_{0}^{1} G^{\star}\left(\frac{1}{2}, s\right) w(s) f\left(s, \int_{0}^{1} G^{\star}(s, r) z(r) g(r, u(r)) d r\right) d s \\
& \geq \int_{1 / 4}^{3 / 4} \sigma G_{1}\left(\frac{1}{2}, s\right) w(s) d s \cdot \lambda_{1} \int_{1 / 4}^{3 / 4} \sigma G_{1}(r, r) z(r) g(r, u(r)) d r \\
& \geq \int_{1 / 4}^{3 / 4} \sigma G_{1}\left(\frac{1}{2}, s\right) w(s) d s \cdot \lambda_{1} \int_{1 / 4}^{3 / 4} \sigma G_{1}(r, r) z(r) \cdot \lambda_{2} u d r \\
& \geq \lambda_{1} \int_{1 / 4}^{3 / 4} \sigma G_{1}\left(\frac{1}{2}, s\right) w(s) d s \cdot \lambda_{2} \int_{1 / 4}^{3 / 4} \sigma G_{1}(r, r) z(r) \cdot m(r)\|u\| d r
\end{aligned}
$$




$$
\begin{aligned}
& \geq \lambda_{1} \int_{1 / 4}^{3 / 4} \sigma G_{1}\left(\frac{1}{2}, s\right) w(s) d s \cdot \lambda_{2} \int_{1 / 4}^{3 / 4} \sigma m(r) G_{1}(r, r) z(r)\|u\| d r \\
& \Longrightarrow\left\|A u\left(\frac{1}{2}\right)\right\| \geq\|u\| .
\end{aligned}
$$

Thus

$$
\|A u\| \geq\left|A u\left(\frac{1}{2}\right)\right| \geq\|u\| .
$$

Setting $\Omega_{2}=\left\{u \in B^{*}:\|u\|<\rho_{2}\right\}$, then $\|A u\| \geq\|u\|$, for $u \in\left(K_{*} \cap \partial \Omega_{2}\right)$.

By the application of part (i) of Theorem 2.11, the operator $A$ has a fixed point in $K \cap\left(\bar{\Omega}_{2} \backslash \Omega_{1}\right)$. This means that the BVP (1) has a positive solution, say $u(t)$, with $e_{2} \leq\|u(t)\| \leq e_{1}$. This completes the proof.

\section{References}

[1] B. Ahmad, J.J. Nieto, Existence results for nonlinear boundary value problems of fractional integrodifferential equations with integral boundary conditions, Boundary Value Problems, 2009 (2009), Article ID 708576, 11pp.; doi:10.1155/2009/708576.

[2] B. Ahmad, J.J. Nieto, A. Alsaed, Existence and uniqueness of solutions for nonlinear fractional differential equations with non-separated type integral boundary conditions, Acta Mathematica Scientia, 31 (2011), 2122-2130.

[3] M.B. Akorede, P.O. Arawomo, Positive solutions for a coupled system of nonlinear second order eigenvalue problems, Int. J. Pure and Applied Mathematics 100, No 1 (2015), 19-28; doi:10.12732/ijpam.v100i1.3.

[4] B. Ahmad, J.J. Nieto, Existence results for a coupled system nonlinear fractional differential equations with three-point boundary conditions, Computers and Mathematics with Appl., 58, No 9 (2009), 1838-1843; doi:10.1016/j.camwa.2009.07.091.

[5] Z. Bai, H. Lu, Positive solutions for boundary value problem of nonlinear fractional differential equation, J. Math. Anal. Appl., 311 (2005), 495-505; doi:10.1016/j.jmaa.2005.02.052.

[6] Z. Bai, On positive solutions of a nonlocal fractional boundary value problem, Nonlinear Analysis: Theory, Methods and Appl., 72, No 2 (2010), 916-924; doi:10.1016/j.na.2009.07.033. 
[7] M. Benchohra, A. Cabada, D. Seba, An existence result for fractional differential equations on Banach spaces, Boundary Value Problems, 2009 (2009), Article ID 628916, 11pp; doi:10.1155/2009/628916.

[8] A. Cabada, Z. Hamdi, Nonlinear fractional differential equations with integral boundary value conditions, Applied Mathematics and Computation, 228 (2014), 251-257; doi:10.1016/j.amc.2013.11.057.

[9] A. Cabada, G. Wang, Positive solutions of nonlinear fractional differential equations with integral boundary value conditions, J. Math. Anal. Appl. 389 (2012), 403-411; doi:10.1016/j.jmaa.2011.11.065.

[10] M. Feng, X. Liu, H. Feng, The existence of positive solutions for a nonlinear fractional differential equation with integral boundary conditions, Adv. Difference Equations, 2011 (2011), Art. ID 546038, 14pp.; doi:10.1155/2011/546038.

[11] J. Henderson, R. Luca, Positive solutions for a system of fractional differential equations with coupled integral boundary conditions, Applied Mathematics and Computation, 249 (2014), 182-197; doi:10.1016/j.amc.2014.10.028.

[12] J. Henderson, R. Luca, A. Tudorache, Positive solutions for systems of coupled fractional boundary value problems, Open J. Applied Sciences, 5 (2015), 600-608; doi:10.4236/ojapps.2015.510059.

[13] J. Henderson, R. Luca, A. Tudorache, Positive solutions for a fractional boundary value problem, Nonlinear Studies, 22, No 1 (2015), 139-151.

[14] J. Jin, X. Liu, M. Jia, Existence of positive solutions for singular fractional differential equations with integral boundary conditions, Electron. J. Diff. Equations, 2012 (2012), No 63, 14pp.

[15] M.A. Krasnosel'skii, Positive Solutions of Operator Equations, Noordhoff, Groningen (1964).

[16] M. Li, Y. Liu, Existence and uniqueness of positive solutions for a coupled system of nonlinear fractional differential equations, Open J. Applied Sciences, 3 (2013), 53-61; doi:10.4236/ojapps.2013.31B1011.

[17] J. Mao, Z. Zhao, N. Xu, On existence and uniqueness of positive solutions for integral boundary value problems, Electron. J. Qual. Theory Diff. Eqs., 2010, No 16 (2010), 8pp. 
[18] K.S. Miller, B. Ross, An Introduction to the Fractional Calculus and Fractional Differential Equations, John Wiley and Sons, New York (1993).

[19] I. Podlubny, Fractional Differential Equations, Ser. Mathematics in Science and Engineering \# 198, Academic Press, San Diego (1999).

[20] S.G. Samko, A.A. Kilbas, O.I. Marichev, Fractional Integrals and Derivatives (Theory and Applications), Gordon and Breach, Switzerland (1993).

[21] X. Su, Boundary value problem for a coupled system of nonlinear fractional differential equations, Applied Mathematics Letters, 22 (2009), 6469; doi:10.1016/j.aml.2008.03.001.

[22] Y. Sun, M. Zhao, Positive solutions for a class of fractional differential equations with integral boundary conditions, Applied Mathematics Letters, 34 (2014), 17-21; doi:10.1016/j.aml.2014.03.008.

[23] J. Wang, H. Xiang, Z. Liu, Positive solutions to nonzero boundary value problem for a coupled system of nonlinear fractional differential equations, Int. J. of Diff. Eqs, 2010 (2010), Art. ID 186928, 12pp.; doi:10.1155/2010/186928.

[24] X. Wang, X. Liu, X. Deng, Existence and nonexistence of positive solutions for fractional integral boundary value problem with two disturbance parameters, Boundary Value Problems, 2015 (2015), No 186; doi:10.1186/s13661-015-0450-1.

[25] Y. Wang, L. Liu, X. Zhang, Y. Wu, Positive solutions for $(n-1,1)$ type singular fractional differential system with coupled integral boundary conditions, Abstr. App. Anal., 2014 (2014), Art. ID 142391, 14pp.; doi:10.1155/2014/142391.

[26] W. Yang, Positive solutions for a coupled system of nonlinear fractional differential equations with integral boundary conditions, Computers and Mathematics with Appl., 63 (2012), 288-297; doi:10.1016/j.camwa.2011.11.021.

[27] K. Zhao, P. Gong, Positive solutions of $m$-point multi-term fractional integral BVP involving time-delay for fractional differential equation, Boundary Value Problems, 2015, No 19 (2015); doi:10.1186/s13661-014-0280-6.

[28] X. Zhang, L. Wang, Q. Sun, Existence of positive solutions for a class of nonlinear fractional differential equation with integral boundary conditions 
and a parameter, Applied Mathematics and Computation, 226 (2014), 708718; doi:10.1016/j.amc.2013.10.089.

[29] H. Zhang, Multiple positive solutions of nonlinear BVPs for differential systems involving integral conditions, Boundary Value Problems, 2014, No 61 (2014); doi:10.1186/1687-2770-2014-61.

[30] C. Zhai, M. Hao, Multiple positive solutions to nonlinear boundary value problem of a system for fractional differential equations, The Scientific World Journal, 2014 (2014), Art. ID 817542, 11pp.; doi:10.1155/2014/817542.

[31] X. Zhang, C. Zhu, Z. Wu, Solvability for a coupled system of fractional differential equations with impulses at resonance, Boundary Value Problem, 2013, No 80 (2013), 23pp.; doi:10.1186/1687-2770-2013-80.

[32] C. Zhu, X. Zhang, Z. Wu, Solvability for a coupled system of fractional differential equations with nonlocal integral boundary conditions, Taiwanese J. Math., 17, No 6 (2013), 2039-2054; doi:10.11650/tjm.17.2013.3174.

[33] Y. Zhao, S. Sun, Z. Han, W. Feng, Positive solutions for a coupled system of nonlinear differential equations of mixed fractional orders, Adv. Difference Equ., 2011, No 10 (2011), 13pp.; doi:10.1186/1687-1847-2011-10. 